Nicolas Verheyen*, Andreas Meinitzer, Martin Robert Grübler, Klemens Ablasser, Ewald Kolesnik, Astrid Fahrleitner-Pammer, Evgeny Belyavskiy, Christian Trummer, Verena Schwetz, Elisabeth Pieske-Kraigher, Jakob Voelkl, loana Alesutan, Cristiana Catena, Leonardo Alberto Sechi, Helmut Brussee, Dirk von Lewinski, Winfried März, Burkert Pieske, Stefan Pilz and Andreas Tomaschitz

\title{
Low-grade inflammation and tryptophan- kynurenine pathway activation are associated with adverse cardiac remodeling in primary hyperparathyroidism: the EPATH trial
}

DOI 10.1515/cclm-2016-1159

Received December 19, 2016; accepted March 24, 2017; previously published online April 22, 2017

\section{Abstract}

Background: Primary hyperparathyroidism (pHPT) is associated with low-grade inflammation, left ventricular hypertrophy and increased cardiovascular mortality, but the association between inflammatory markers and parameters of adverse cardiac remodeling is unknown. We investigated the relationship between C-reactive protein (CRP), the essential amino acid tryptophan and its proinflammatory derivatives kynurenine and quinolinic acid (QUIN) with echocardiographic parameters.

Methods: Cross-sectional baseline data from the "Eplerenone in Primary Hyperparathyroidism" trial were analyzed. Patients with any acute illness were excluded. We assessed associations between CRP, serum levels of tryptophan, kynurenine and QUIN and left ventricular mass index (LVMI), left atrial volume index (LAVI) and E/e'.

\footnotetext{
*Corresponding author: Nicolas Verheyen, Department of Cardiology, Medical University of Graz, Auenbruggerplatz 15, 8036 Graz, Austria, Phone: +43 (316)385-30173, Fax: +43 (316)385-13733, E-mail: nicolas.verheyen@medunigraz.at

Andreas Meinitzer: Clinical Institute of Medical and Chemical Laboratory Diagnostics, Medical University of Graz, Graz, Austria Martin Robert Grübler: Department of Cardiology, Swiss Cardiovascular Center Bern, Bern University Hospital, Bern, Switzerland

Klemens Ablasser, Ewald Kolesnik, Helmut Brussee and Dirk von Lewinski: Department of Cardiology, Medical University of Graz, Graz, Austria

Astrid Fahrleitner-Pammer, Christian Trummer and Verena Schwetz: Division of Endocrinology and Diabetology, Department of Internal Medicine, Medical University of Graz, Graz, Austria Evgeny Belyavskiy, Elisabeth Pieske-Kraigher, Jakob Voelkl, Ioana Alesutan and Burkert Pieske: Medizinische Klinik mit Schwerpunkt Kardiologie, Campus Virchow-Klinikum, CharitéUniversitaetsmedizin Berlin, Berlin, Germany
}

Results: Among 136 subjects with pHPT (79\% females), 100 (73\%) had arterial hypertension and the prevalence of left ventricular hypertrophy was 52\%. Multivariate linear regression analyses with LVMI, LAVI and E/e' as respective dependent variables, and C-reactive protein and tryptophan, kynurenine and QUIN as respective independent variables were performed. Analyses were adjusted for age, sex, blood pressure, parathyroid hormone, calcium and other cardiovascular risk factors. LVMI was independently associated with CRP (adjusted $\beta$-coefficient $=0.193$, $\mathrm{p}=0.030)$ and QUIN $(\beta=0.270, \mathrm{p}=0.007)$, but not kynurenine. LAVI was related with CRP $(\beta=0.315, p<0.001)$, kynurenine $(\beta=0.256, p=0.005)$ and QUIN $(\beta=0.213$, $p=0.044) . E / e^{\prime}$ was related with kynurenine $(\beta=0.221$, $\mathrm{p}=0.022)$ and QUIN $(\beta=0.292, \mathrm{p}=0.006)$. Tryptophan was not associated with any of the remodeling parameters.

[Correction added after online publication (22 April 2017: The sentence "Among 136 subjects with pHPT (79\% females), 100 (73\%) had left ventricular hypertrophy."

Cristiana Catena and Leonardo Alberto Sechi: Hypertension Unit, Internal Medicine, Department of Experimental and Clinical Medical Sciences, University of Udine, Udine, Italy

Winfried März: Clinical Institute of Medical and Chemical Laboratory Diagnostics, Medical University of Graz, Graz, Austria; Synlab Academy, Synlab Services LLC, Mannheim, Germany; and Medical Clinic V (Nephrology, Hypertensiology, Endocrinology), Medical Faculty Mannheim, Ruperto Carola University Heidelberg, Mannheim, Germany

Stefan Pilz: Division of Endocrinology and Diabetology, Department of Internal Medicine, Medical University of Graz, Graz, Austria; and Department of Health Sciences and the $\mathrm{EMGO}^{+}$Institute, VU University Amsterdam, The Netherlands Andreas Tomaschitz: Bad Gleichenberg Clinic, Bad Gleichenberg, Austria; and Medical Clinic V (Nephrology, Hypertensiology, Endocrinology), Medical Faculty Mannheim, Ruperto Carola University Heidelberg, Mannheim, Germany 
was corrected to "Among 136 subjects with pHPT (79\% females), $100(73 \%)$ had arterial hypertension and the prevalence of left ventricular hypertrophy was 52\%.”]

Conclusions: Cardiac remodeling is common in pHPT and is associated with low-grade inflammation and activation of the tryptophan-kynurenine pathway. The potential role of kynurenine and QUIN as cardiovascular risk factors may be further investigated in future studies.

Keywords: cardiac remodeling; inflammation; kynurenine; primary hyperparathyroidism; quinolinic acid; tryptophan.

\section{Introduction}

Primary hyperparathyroidism (pHPT), the third most common endocrine disorder, is associated with an increased prevalence of left ventricular (LV) hypertrophy [1], LV stiffening and risk of cardiovascular mortality [2, 3]. Although the prevalence of arterial hypertension in pHPT is high, LV hypertrophy is often diagnosed in subjects without arterial hypertension, suggesting that further mechanisms may contribute to adverse cardiac remodeling [4]. In general, low-grade inflammation plays a pivotal role in the development of cardiovascular disease, and recent evidence suggests a mediating role of metabolites of the essential amino acid tryptophan [5]. Under inflammatory conditions, tryptophan is enzymatically degraded to kynurenine, hydroxy-kynurenine and quinolinic acid (QUIN), see Figure 1. Kynurenines further mediate interferon- $\gamma$ induced oxidative stress leading to endothelial cell apoptosis and endothelial dysfunction in vitro and in vivo [6]. Via promoting oxidative stress, also QUIN has been shown to induce cell death $[9,10]$. Derivatives of

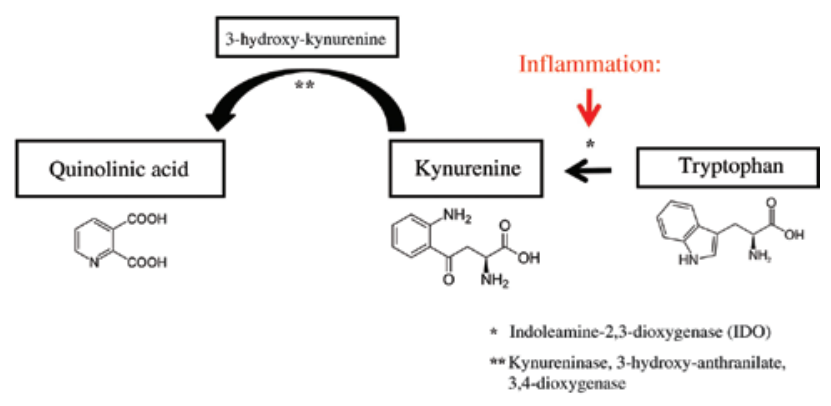

Figure 1: Schematic overview on the tryptophan-kynurenine pathway.

Under inflammatory conditions, tryptophan is metabolized to kynurenine via indoleamine-2,3-dioxygenase-1 (ID01) [6]. Further enzymatic degradation to 3-hydroxykynurenine and quinolinic acid occurs systemically, mainly in macrophages $[7,8]$. IFN, interferon. the tryptophan-kynurenine pathway were linked with cardiovascular disease in dialysis patients [11, 12] and were shown to predict myocardial infarction in subjects referred to coronary angiography [13]. Also in individuals without coronary heart disease, circulating kynurenine levels were an independent predictor of acute coronary events [14].

Several studies demonstrated increased levels of inflammatory markers in subjects with pHPT when compared to healthy individuals $[15,16]$. In a small $\mathrm{pHPT}$ cohort, inflammatory markers were partly related with cardiac function [17]. Of note, an increase in kynurenine-to-tryptophan ratio has been reported in subjects with pHPT [18]. However, the role of metabolites of the tryptophan-kynurenine pathway for cardiac remodeling in PHPT has not yet been investigated. In light of this gap in evidence, we aimed to test the hypothesis of a direct link between parameters of the tryptophan-kynurenine pathway, inflammation and echocardiographic parameters of cardiac remodeling in pHPT, applying a novel and recently validated method to determine QUIN concentrations in serum [19].

\section{Materials and methods}

\section{Patients and setting}

Cross-sectional data from screening participants of the singlecenter, randomized, placebo-controlled, double-blind, parallel-arm "Eplerenone in Primary Hyperparathyroidism” (EPATH) Study were used in the present analyses. Details on the study design and setting have been previously reported [20, 21]. Main inclusion criteria for trial participation were age over 18 years and biochemical diagnosis of pHPT. Main exclusion criteria included any acute illness and life expectancy $<1$ year as judged by an experienced clinician. Screening procedures included blood sampling, transthoracic echocardiography and 24-h ambulatory blood pressure monitoring (ABPM).

For the present analyses, we enrolled all 155 screening participants with a confirmed biochemical diagnosis of pHPT. Exclusion of 19 subjects with unsuccessful ABPM $(n=16)$ or unavailable echocardiography $(n=3)$ yielded a sample size of 136 subjects. From subjects with available echocardiography, left ventricular mass index (LVMI) or left atrial volume index (LAVI) could not be determined in 13 and one subjects, respectively, because of inappropriate anatomical condition or insufficient image quality. The flowchart of subjects included in the present analyses is provided in Supplemental Figure 1. All patients provided written informed consent. The study was approved by the local Ethics Committee and complies with the good clinical practice guidelines and the Declaration of Helsinki.

\section{Laboratory, echocardiography and ABPM}

Blood samplings were performed during the morning (07:00 AM11:00 AM) after an overnight fast. Patients remained in the seated 
position before blood sampling for at least $10 \mathrm{~min}$. All samples were kept at room temperature before analyses, except for those that were used to measure parathyroid hormone (PTH), which were kept at $4^{\circ} \mathrm{C}$. A prespecified volume of blood samples was centrifuged, aliquoted and frozen at $-80^{\circ} \mathrm{C}$. Both tryptophan and kynurenine were determined in serum using high-performance liquid chromatography with a simultaneous ultraviolet and fluorimetric detection system [22]. Within-day coefficients of variation (CVs) at different concentrations are in the range of $1.7 \%$ to $4.3 \%$ for kynurenine and $0.7 \%$ to $2.9 \%$ for tryptophan. The between-day CVs are $2.0 \%$ to $5.4 \%$ and $6.3 \%$ to $9.3 \%$, respectively. QUIN was determined in serum using a liquid chromatography tandem mass spectrometry method [19]. Within-day CVs are 4.5\% (225 nmol/L) and 1.2\% (725 nmol/L), and between-day CVs are $7.2 \%(235 \mathrm{nmol} / \mathrm{L})$ and $6.3 \%(752 \mathrm{nmol} / \mathrm{L})$. Other laboratory methods have been previously described [21, 23].

Echocardiographic examinations were performed with a Vivid seven or Vivid nine (GE Healthcare, Chalfont St Giles, UK). All images and recorded loops were digitally saved for later analyses in a central core lab (Echocardiography CoreLab, Department of Cardiology, Medical University of Graz, Graz, Austria). All loops and images were analyzed by one investigator (EB) who was blinded to individual participant data. A validated continuous ABPM portable device (MobilO-Graph, I.E.M. GmbH, Stolberg, Germany) was applied to perform ABPM. Mean nocturnal blood pressure was calculated as the mean of all valid measurements between 01:00 and 06:00 AM. Further details on laboratory, echocardiographic and ABPM methods have been previously reported [20, 21, 23].

\section{Definitions}

PHPT was defined as (1) hypercalcemia (total serum albumin-adjusted calcium $>2.55 \mathrm{mmol} / \mathrm{L}$ ) in concomitance with inappropriately high PTH of $>46 \mathrm{pg} / \mathrm{mL}$ or (2) hyperparathyroidemia (PTH $>65 \mathrm{pg} / \mathrm{mL}$ ), albumin-adjusted plasma calcium above the median of the reference range $(>2.35 \mathrm{mmol} / \mathrm{L})$ and plasma-ionized calcium within normal ranges, in the absence of advanced chronic kidney disease (estimated glomerular filtration rate $[\mathrm{eGFR}]>40 \mathrm{~mL} / \mathrm{min} / 1.73 \mathrm{~m}^{2}$ ) or 25 -hydroxy vitamin D [25(OH)D] deficiency [25(OH)D $<20 \mathrm{ng} / \mathrm{mL}]$, as potential causes for secondary HPT [21]. LV mass (LVM) was calculated according to the modified Devereux formula and was indexed for body surface area to compute LVMI [24]. Left atrial volume (LAV) was calculated using the biplane area-length method and was indexed for body surface area to compute LAVI [24]. LV hypertrophy and left atrial enlargement were determined according to international guidelines [24]. Left ventricular ejection fraction (LVEF) was assessed by biplane Simpson method of disks [24]. E/e' as a measure of LV stiffness was averaged from measurements of both the septal and the lateral mitral ring, and $\mathrm{E} / \mathrm{e}^{\prime}>15$ was considered elevated [25]. Arterial hypertension was defined as a mean 24-h ABPM blood pressure $\geq 130 / 80 \mathrm{mmHg}$ or as regular use of at least one antihypertensive drug for the treatment of arterial hypertension. Calcium was adjusted for hypoalbuminemia as previously reported [21]. EGFR was calculated using the chronic kidney disease epidemiology collaboration equation.

\section{Statistical methods}

Continuous variables were given as the mean \pm standard deviation or as the median with interquartile range, as appropriate. Categorical variables were described as numbers and percentages and were illustrated in histograms. The distribution of continuous parameters and their residuals were evaluated by test of Kolmogorov-Smirnov, kurtosis, skewness, concordance between the mean and median and visual inspection. Nonnormally distributed variables were logarithmized, before use in parametrical procedures. Associations between baseline characteristics were assessed using Pearson correlation or $\chi^{2}$-test, as appropriate. Correlations were illustrated in scatterplots using a quadratic function. Attempting to understand the impact of potential confounders on the tryptophan-kynurenine cascade, we set up three multivariate stepwise linear regression analyses with tryptophan, kynurenine or QUIN, as respective dependent variables. Covariates were age, sex, diabetes mellitus (yes/no), mean nocturnal systolic blood pressure, antihypertensive drugs (number), eGFR, body mass index (BMI), total serum cholesterol, active smoking (yes/no), PTH, adjusted plasma calcium and C-reactive protein (CRP). Moreover, CRP, tryptophan, kynurenine and QUIN were correlated with echocardiographic parameters of cardiac remodeling (LVMI, LAVI, E/e'), LVEF and N-terminal pro-brain B-type natriuretic peptide (NT-proBNP) in multivariate stepwise linear regression analyses using covariates as described above, without CRP (model 1). To assess the potentially modifying role of inflammation, we adjusted for CRP in an additional model (model 2). Analyses of variance (ANOVA) and covariance (ANCOVA) with CRP, tryptophan, kynurenine or QUIN as dependent variables and LVH (yes/no), LA enlargement (yes/no) or E/e' $>15$ as the respective fixed factors were performed, with parameters of model one as covariates for ANCOVA.

For statistical analyses, we used SPSS 23.0 (SPSS, Inc, Chicago, IL, USA). A two-sided $\mathrm{p}$-value $<0.05$ was considered statistically significant.

\section{Results}

Mean age was $67 \pm 10$ years and 107 participants (79\%) were females. The prevalence of LV hypertrophy was 52\% and 100 subjects (73\%) had arterial hypertension. Median E/e' (average) was 10 ([10-15]) and 19 subjects (14\%) had $\mathrm{E} / \mathrm{e}^{\prime}>15$. Circulating concentrations of tryptophan, kynurenine and QUIN were $56 \pm 10 \mu \mathrm{mol} / \mathrm{L}, 2.79 \pm 0.76 \mu \mathrm{mol} / \mathrm{L}$ and 505 (404-640) nmol/L, respectively. Further baseline characteristics are provided in Table 1 . PTH was significantly correlated with QUIN (Pearson $\mathrm{r}=0.173, \mathrm{p}=0.044$ ), but not with CRP, tryptophan or kynurenine. Univariate correlations between CRP and derivatives of tryptophan with cardiac remodeling parameters are shown in Table 2.

\section{Parameters associated with tryptophan, kynurenine and QUIN concentrations in multivariate analyses}

Tryptophan was significantly associated with female sex $(\beta=0.279, p=0.003)$ and BMI $(\beta=0.234, p=0.032)$. Covariates showing the strongest associations with kynurenine were eGFR $(\beta=-0.552, p<0.001)$, tryptophan $(\beta=0.431$, $\mathrm{p}<0.001)$, cholesterol $(\beta=-0.215, \mathrm{p}=0.003)$ and CRP $(\beta=0.191, p=0.010)$. QUIN was independently related with 
Table 1: Baseline characteristics of the studied cohort.

\begin{tabular}{lr}
\hline Age, years & $67 \pm 10$ \\
Females, $\mathrm{n}(\%)$ & $107(78 \%)$ \\
BMI, $\mathrm{kg} / \mathrm{m}^{2}$ & $28 \pm 5$ \\
Estimated glomerular filtration rate (CKDEPI), & $77 \pm 16$ \\
$\mathrm{~mL} / \mathrm{min} / 1.73 \mathrm{~m}^{2}$ & \\
CRP, $\mathrm{mg} / \mathrm{L}$ & $0.15(0.6-2.7)$ \\
Active smokers, $\mathrm{n}(\%)$ & $19(14 \%)$ \\
Diabetes mellitus, $\mathrm{n}(\%)$ & $13(10 \%)$ \\
PTH (1-84), $\mathrm{pg} / \mathrm{mL}$ & $99(82-124)$ \\
$25-$ Hydroxyvitamin D, $\mathrm{ng} / \mathrm{mL}$ & $32(26-40)$ \\
Adjusted plasma calcium, $\mathrm{mmol} / \mathrm{L}$ & $2.63 \pm 0.15$ \\
Tryptophan-kynurenine pathway & \\
Tryptophan, $\mu$ mol/L & $55.8 \pm 10.1$ \\
Kynurenine, $\mu$ mol/L & $2.79 \pm 0.76$ \\
Quinolinic acid, $\mathrm{nmol} / \mathrm{L}$ & $505(404-640)$ \\
Cardiovascular parameters & \\
Left ventricular ejection fraction, \% & $63(59-68)$ \\
E/e' & $10(8-13)$ \\
Left ventricular mass index, $\mathrm{g} / \mathrm{m}^{2}$ & $103(81-123)$ \\
Left atrial volume index, $\mathrm{mL} / \mathrm{m}^{2}$ & $30(23-36)$ \\
Mean nocturnal systolic blood pressure, $\mathrm{mmHg}$ & $116(106-128)$ \\
Mean nocturnal diastolic blood pressure, $\mathrm{mmHg}$ & $69 \pm 11$ \\
Arterial hypertension, $\mathrm{n}(\%)$ & $100(73 \%)$ \\
Antihypertensive medication, $\mathrm{n}(\%)$ & $85(64 \%)$ \\
\hline
\end{tabular}

kynurenine $(\beta=-0.525, p<0.001)$, cholesterol $(\beta=-0.275$, $\mathrm{p}=0.003)$, eGFR $(\beta=-0.257, \mathrm{p}=0.001)$, age $(\beta=0.227$, $\mathrm{p}=0.001)$ and CRP $(\beta=0.198, \mathrm{p}=0.001)$, as illustrated in more detail in Table 3.

\section{Relationships between CRP, tryptophan, kynurenine, QUIN and parameters of cardiac structure and function in multivariate linear regression analyses}

CRP was independently related with LVMI $(\beta$-coefficient $=-0.193, p=0.030)$ and LAVI $(\beta=-0.315$, $p<0.001)$ and NT-proBNP $(\beta=-0.158, p=0.043)$, but not with LVEF or E/e'. Tryptophan was not correlated with any of the echocardiographic parameters. Kynurenine showed independent correlations with LAVI (model 1: $\beta=0.256, p=0.005)$ and $E / e^{\prime}(\beta=0.221, p=0.022)$, but not with LVMI, LVEF or NT-proBNP. QUIN was independently correlated with LVMI (model 1: $\beta=0.270$, $p=0.007$ ), LAVI (model 1: $\beta=0.213, p=0.044$ ), $E / e^{\prime}$ (model 1: $\beta=0.292, p=0.006)$ and NT-proBNP $(\beta=0.192$, $p=0.037$, but not with LVEF. Adjustment for CRP blunted the significance of correlations between kynurenine and $\mathrm{E} / \mathrm{e}^{\prime}$ as well as of QUIN with both LAVI and NT-proBNP, respectively. These results are shown in Table 4. Figure 2 shows scatterplots depicting correlations between kynurenine and QUIN with LVMI, LAVI and $\mathrm{E} / \mathrm{e}^{\prime}$, respectively.

\section{Associations between CRP, tryptophan, kynurenine, QUIN and conditions of adverse cardiac remodeling in multivariate ANCOVAs}

Concentrations of both CRP and tryptophan did not differ significantly by LVH, LA enlargement or elevated $\mathrm{E} / \mathrm{e}^{\prime}$, respectively. Kynurenine was significantly higher in patients with LA enlargement than in those without [adjusted mean, 2.96 (95\% CI, 2.81-3.11) $\mu \mathrm{mol} / \mathrm{L}$ vs. 2.58 (2.40-2.76) $\mu \mathrm{mol} / \mathrm{L}, \mathrm{p}=0.004]$. Subjects with LVH exhibited higher concentrations of QUIN compared to those without LVH [adjusted mean 580 (529-631) nmol/L vs. $502(529-630) \mathrm{nmol} / \mathrm{L}, \mathrm{p}=0.047]$. Subjects with $\mathrm{E} / \mathrm{e}^{\prime}>15$ had significantly higher levels of QUIN than those with E/e' $<15$ [adjusted mean 682 (592-772) nmol/L vs. 538 (502-573) nmol/L, p=0.022]. Results for kynurenine and QUIN are shown in Table 5. The frequency of LVH and LA enlargement across QUIN tertiles is illustrated in Figure 3.

Table 2: Univariate correlations between CRP and tryptophan derivatives with parameters of cardiac structure and function.

\begin{tabular}{|c|c|c|c|c|c|c|c|c|c|}
\hline & \multirow[t]{2}{*}{ n } & \multicolumn{2}{|r|}{ CRP } & \multicolumn{2}{|c|}{ Tryptophan } & \multicolumn{2}{|c|}{ Kynurenine } & \multicolumn{2}{|c|}{ Quinolinic acic } \\
\hline & & $r$ & p-Value & $r$ & p-Value & $\mathbf{r}$ & p-Value & $r$ & p-Value \\
\hline LVMI & 136 & 0.202 & 0.025 & -0.005 & 0.599 & 0.272 & 0.002 & 0.383 & $<0.001$ \\
\hline LAVI & 135 & 0.273 & 0.001 & 0.022 & 0.798 & 0.369 & $<0.001$ & 0.336 & $<0.001$ \\
\hline LVEF & 123 & -0.150 & 0.082 & -0.063 & 0.469 & -0.178 & 0.038 & -0.153 & 0.076 \\
\hline E/e' (average) & 136 & 0.091 & 0.290 & -0.026 & 0.766 & 0.159 & 0.064 & 0.321 & $<0.001$ \\
\hline NT-proBNP & 136 & 0.090 & 0.300 & -0.212 & 0.013 & 0.232 & 0.007 & 0.420 & $<0.001$ \\
\hline
\end{tabular}

CRP, C-reactive protein; LVMI, left ventricular mass index; LAVI, left atrial volume index; LVEF, left ventricular ejection fraction; NT-proBNP, $\mathrm{N}$-terminal pro-brain B-type natriuretic peptide. Correlations were assessed using Pearson correlation analysis. Significant correlations are highlighted in bold. 
Table 3: Parameters associated with tryptophan, kynurenine and quinolinic acid in multivariate models.

\begin{tabular}{|c|c|c|c|c|c|c|}
\hline & \multicolumn{2}{|c|}{ Tryptophan } & \multicolumn{2}{|c|}{ Kynurenine } & \multicolumn{2}{|c|}{ Quinolinic acid } \\
\hline & $\beta$ & p-Value & $\beta$ & p-Value & $\boldsymbol{\beta}$ & p-Value \\
\hline Age & -0.133 & 0.217 & -0.101 & 0.216 & 0.227 & 0.001 \\
\hline Male gender & 0.279 & 0.003 & 0.146 & 0.042 & -0.123 & 0.036 \\
\hline Diabetes mellitus & -0.084 & 0.370 & -0.036 & 0.610 & -0.071 & 0.218 \\
\hline Mean nocturnal SBP & 0.019 & 0.831 & -0.081 & 0.238 & -0.042 & 0.445 \\
\hline Number of antihypertensive drugs & -0.071 & 0.521 & -0.143 & 0.089 & 0.030 & 0.659 \\
\hline eGFR (CKDEPI) & 0.109 & 0.311 & -0.552 & $<0.001$ & -0.257 & 0.001 \\
\hline BMI & 0.234 & 0.032 & 0.155 & 0.065 & -0.031 & 0.651 \\
\hline Total cholesterol & 0.109 & 0.246 & -0.215 & 0.003 & -0.275 & $<0.001$ \\
\hline Smoking & -0.144 & 0.137 & -0.111 & 0.136 & 0.009 & 0.885 \\
\hline PTH & -0.124 & 0.182 & 0.035 & 0.617 & 0.056 & 0.327 \\
\hline Calcium & 0.014 & 0.881 & -0.123 & 0.085 & 0.069 & 0.238 \\
\hline CRP & -0.112 & 0.246 & 0.191 & 0.010 & 0.198 & 0.001 \\
\hline Tryptophan & - & - & 0.431 & $<0.001$ & -0.020 & 0.752 \\
\hline Kynurenine & - & - & - & - & 0.525 & $<0.001$ \\
\hline Adjusted $\mathrm{R}^{2}$ & \multicolumn{2}{|c|}{0.088} & \multicolumn{2}{|c|}{0.477} & \multicolumn{2}{|c|}{0.662} \\
\hline
\end{tabular}

SBP, systolic blood pressure; eGFR, estimated glomerular filtration rate (CKDEPI formula); BMI, body mass index; PTH, parathyroid hormone; CRP, C-reactive protein. Determinants of tryptophan, kynurenine as well as quinolinic acid (dependent variables) were assessed in three separate multivariate stepwise linear regression analyses. All covariates of the models are shown. Significant correlations are highlighted in bold. $\beta$ represents adjusted correlation coefficient.

Table 4: Multivariate correlations between CRP and tryptophan derivatives with parameters of cardiac structure and function.

\begin{tabular}{|c|c|c|c|c|c|c|c|c|}
\hline \multirow[t]{2}{*}{ Dependent variable } & \multicolumn{2}{|r|}{ CRP } & \multicolumn{2}{|c|}{ Tryptophan } & \multicolumn{2}{|c|}{ Kynurenine } & \multicolumn{2}{|c|}{ Quinolinic acid } \\
\hline & $\beta$ & p-Value & $\boldsymbol{\beta}$ & p-Value & $\boldsymbol{\beta}$ & p-Value & $\boldsymbol{\beta}$ & p-Value \\
\hline \multicolumn{9}{|c|}{ Left ventricular mass index, $\mathrm{n}=123$} \\
\hline Model 1 & 0.193 & 0.030 & -0.004 & 0.962 & 0.156 & 0.092 & 0.270 & 0.007 \\
\hline Model 2 & - & - & 0.015 & 0.856 & 0.124 & 0.184 & 0.222 & 0.035 \\
\hline \multicolumn{9}{|c|}{ Left atrial volume index, $n=135$} \\
\hline Model 1 & 0.315 & $<0.001$ & 0.014 & 0.869 & 0.256 & 0.005 & 0.213 & 0.044 \\
\hline Model 2 & - & - & 0.043 & 0.591 & 0.302 & 0.001 & 0.103 & 0.338 \\
\hline \multicolumn{9}{|c|}{ Left ventricular ejection fraction, $n=136$} \\
\hline Model 1 & -0.119 & 0.226 & 0.018 & 0.840 & -0.117 & 0.264 & -0.187 & 0.106 \\
\hline Model 2 & - & - & 0.007 & 0.940 & -0.100 & 0.345 & -0.158 & 0.197 \\
\hline \multicolumn{9}{|l|}{$E / e^{\prime}, n=136$} \\
\hline Model 1 & 0.062 & 0.499 & 0.098 & 0.252 & 0.221 & 0.022 & 0.292 & 0.006 \\
\hline Model 2 & - & - & 0.105 & 0.222 & 0.031 & 0.732 & 0.300 & 0.008 \\
\hline \multicolumn{9}{|l|}{ NT-proBNP, $n=136$} \\
\hline Model 1 & 0.158 & 0.043 & -0.067 & 0.362 & 0.139 & 0.097 & 0.192 & 0.037 \\
\hline Model 2 & - & - & -0.052 & 0.475 & 0.116 & 0.167 & 0.147 & 0.128 \\
\hline
\end{tabular}

NT-proBNP, N-terminal pro-brain B-type natriuretic peptide. Multivariate regression analyses with adjustment for age, sex, diabetes mellitus (yes/no), mean nocturnal systolic blood pressure, antihypertensive drugs (number), estimated glomerular filtration rate (CKDEPI), BMI, serum total cholesterol, active smoking (yes/no), parathyroid hormone (PTH) and adjusted calcium (model 1). Model 2 additionally comprises CRP as covariate. Significant correlations are highlighted in bold. $\beta$ represents adjusted $\beta$-coefficient.

\section{Discussion}

In a cohort of subjects with pHPT, we found that (1) circulating concentrations of kynurenine and QUIN, but not tryptophan, were independently and directly related with CRP; (2) CRP was independently associated with LVMI and LAVI; (3) kynurenine was independently related with LAVI, left atrial enlargement and E/e'; and (4) QUIN was independently associated with LAVI, LVMI, LV hypertrophy, E/e' and NTproBNP. These data provide novel evidence that inflammation and acceleration of the tryptophan-kynurenine pathway link directly with adverse cardiac remodeling in pHPT. 
A

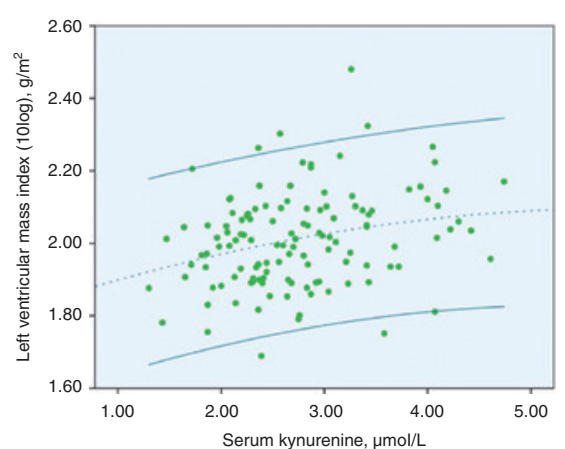

D

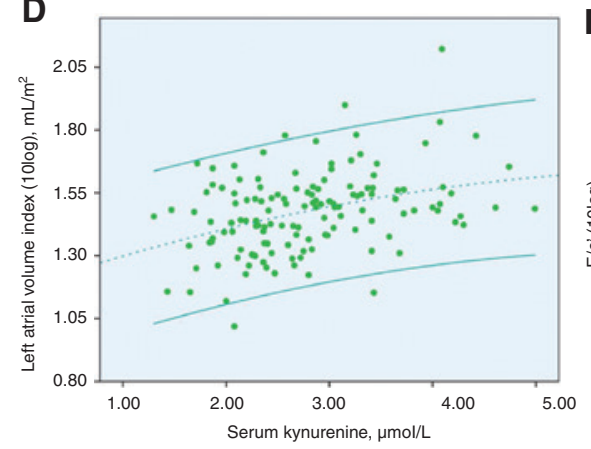

B

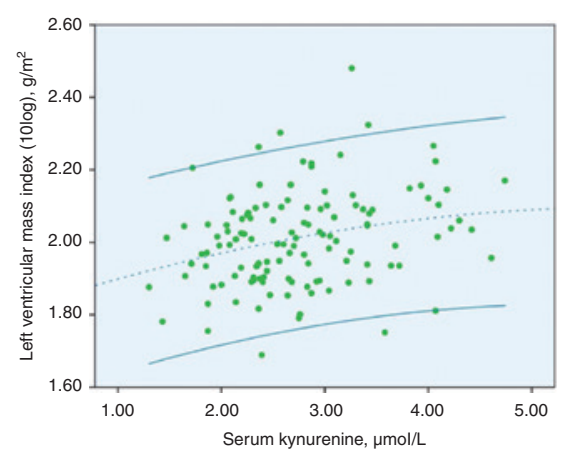

E

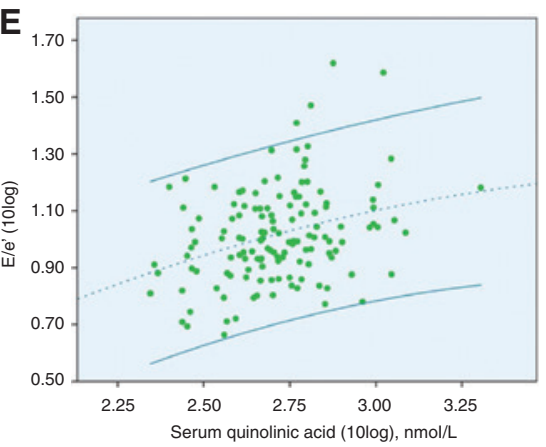

C
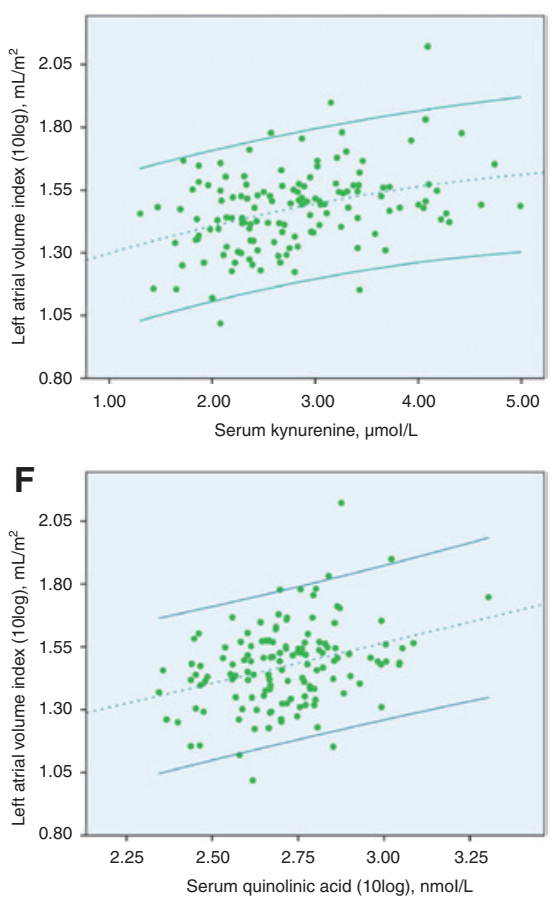

Figure 2: Scatterplots showing relationships between tryptophan derivatives and cardiac remodeling parameters.

Associations of serum kynurenine with (A) left ventricular mass index, (B) E/e' and (C) left atrial volume index, as well as of quinolinic acid with (D) left ventricular mass index, (E) E/ $e^{\prime}$ and (F) left atrial volume index are illustrated. A quadratic function was used for illustration; top and bottom lines indicate $95 \%$ confidence interval margins. Correlations were analyzed using Pearson correlation coefficient.

Table 5: Associations of kynurenine and quinolinic acid with cardiac phenotypes of cardiac remodeling.

\begin{tabular}{|c|c|c|c|c|}
\hline & \multicolumn{2}{|c|}{ Kynurenine, $\mu \mathrm{mol} / \mathrm{L}$} & \multicolumn{2}{|c|}{ Quinolinic acid, nmol/L } \\
\hline & Adjusted mean $(95 \% \mathrm{Cl})$ & p-Value & Adjusted mean $(95 \% \mathrm{Cl})$ & p-Value \\
\hline \multicolumn{5}{|l|}{ LVH } \\
\hline Yes $(n=64)$ & $2.83(2.66-2.99)$ & 0.272 & $580(529-631)$ & 0.047 \\
\hline No $(n=59)$ & $2.69(2.52-2.86)$ & & $502(529-630)$ & \\
\hline \multicolumn{5}{|l|}{ LA enlargement } \\
\hline Yes $(n=77)$ & $2.96(2.81-3.11)$ & 0.004 & $570(524-617)$ & 0.235 \\
\hline No $(n=58)$ & $2.58(2.40-2.76)$ & & $541(486-595)$ & \\
\hline \multicolumn{5}{|l|}{ Elevated $\mathrm{E} / \mathrm{e}^{\prime}$} \\
\hline Yes $(n=19)$ & $3.01(2.71-3.31)$ & 0.122 & $682(592-772)$ & 0.022 \\
\hline No $(n=117)$ & $2.76(2.64-2.88)$ & & $538(502-573)$ & \\
\hline
\end{tabular}

LVH, left ventricular hypertrophy; LA, left atrial; Cl, confidence interval. p-Values for differences of kynurenine or quinolinic acid (10 log) between groups. ANCOVAs with kynurenine or quinolinic acid as dependent variables and LVH (yes/no), LA enlargement (yes/no) or E/e' $>15$ (yes/no) as the respective fixed factors. Covariates include age, sex, diabetes mellitus (yes/no), mean nocturnal systolic blood pressure, antihypertensive drugs (number), estimated glomerular filtration rate (CKDEPI), BMI, serum total cholesterol, active smoking (yes/no), PTH and adjusted calcium.

Low-grade inflammation is well established as a crucial mediator of adverse cardiac remodeling. Markers of chronic low grade inflammation are increased in failing human hearts independently of the underlying cause of heart failure [26]. Furthermore, high levels of CRP predict new incident heart failure [27], incident coronary heart disease and vascular mortality, even in subjects without cardiovascular disease at baseline [28]. Patients with pHPT are at increased risk of LV hypertrophy and death from a cardiovascular cause $[1,3]$, but underlying mechanisms remain incompletely understood. Previous studies consistently demonstrated increased levels of inflammatory markers, such as CRP, interferon- $\gamma$ or the kynurenine-to-tryptophan ratio, in subjects with pHPT when 

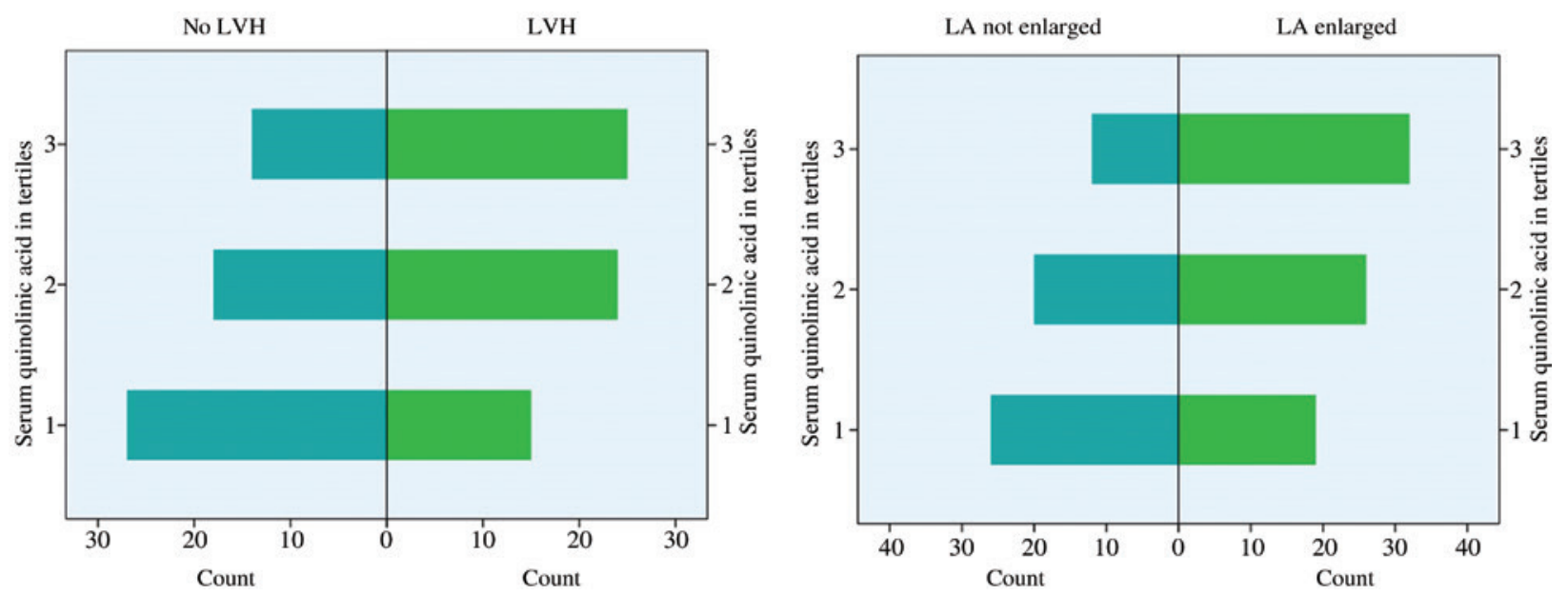

Figure 3: Histograms of left ventricular hypertrophy and left atrial enlargement by quinolinic acid tertiles.

Echocardiographic variables were calculated according to international guidelines [24]. Differences across tertiles were analyzed for significance using $\chi^{2}$-test and $p$-value for linear-by-linear association. LVH, left ventricular hypertrophy; LA, left atrium.

compared to healthy controls $[15,16,18]$, and one study identified an univariate association between inflammatory markers and cardiac function [17]. Our observation of an independent association between CRP with LVMI, LAVI and NT-proBNP, respectively, further supports the hypothesis that inflammation may play a critical role for cardiovascular disease in pHPT.

The present study is the first to provide clinical evidence on parameters associated with tryptophan metabolites in a clinical setting, as well as on an association between tryptophan derivatives and cardiac remodeling. Degradation of tryptophan to kynurenine via ubiquitously expressed IDO1 is accelerated upon inflammatory stimulus. This is reflected by our observation that CRP was independently associated with kynurenine and QUIN in multivariate analyses. Importantly, these derivatives may mediate inflammation-induced cardiovascular disease. Wang and colleagues demonstrated that interferon- $\gamma$ induced degradation of tryptophan to kynurenine in mice [6]. In the same study, kynurenines induced endothelial cell apoptosis and dysfunction, probably via an increased production of superoxide anions. In another study, IDO activity was described in periaortic fat tissue and both kynurenine and QUIN reduced vasopressor induced maximal aortic contraction although the clinical relevance of these findings for cardiovascular disease is unclear [29]. QUIN acts as an agonist on the $N$-methyl$\mathrm{D}$-aspartate (NMDA) receptor [7], which is predominantly expressed in neuronal tissues, but also in cardiomyocytes where a contributing role to oxidative stress, mitochondrial dysfunction, apoptosis and autophagy processes was demonstrated [30, 31]. Increasing evidence suggests that QUIN is relevant for inflammatory processes and oxidative stress [32]. Yet, most evidence on pro-inflammatory and cytotoxic effects of QUIN is derived from experimental studies on neuronal cells. QUIN infiltrations in rat striatal cells induced cellular damage mediated by superoxide anion production [9]. Other explanatory mechanisms for cytotoxic effects of QUIN include intracellular calcium overload by impairment of the sarco/endoplasmic reticulum $\mathrm{Ca}^{2+}$-ATPase (SERCA) activity [33], an enzyme that also crucially regulates calcium handling in cardiomyocytes. QUIN is produced upon inflammatory stimulation in the systemic circulation, besides the central nervous system, where macrophages are considered as the major source [8]. In conditions of increased QUIN concentrations, such as in advanced chronic kidney disease, QUIN was independently associated with carotid intima media thickness [34]. Clinical data on associations of QUIN with cardiac remodeling are, however, lacking, mainly due to the unavailability of laboratory assays to determine QUIN in serum. For the present study, a novel liquid chromatography-mass spectrometry method was applied that has recently been validated [19]. Our observations of an independent link between kynurenine and QUIN with echocardiographic parameters of cardiac remodeling support previous studies that indicated a relevant role for cardiovascular disease. Moreover, our data are consistent with previous longitudinal analyses. Kynurenine or the kynurenine-to-tryptophan ratio were reported as independent determinants of cardiovascular disease events in high-risk cohorts as well as in patients without coronary heart disease at baseline [11-14].

Organ-specific tryptophan degradation is also induced by indoleamine-2,3-dioxygenase-2 or tryptophan2,3-dioxygenase [35]. Of note, the latter can be induced by 
cortisol, and increased cortisol levels have been reported in pHPT patients $[36,37]$. Therefore, cortisol may trigger activation of the tryptophan-kynurenine pathway in pHPT, in addition to low-grade inflammation.

QUIN showed stronger and more robust associations with cardiac remodeling parameters than kynurenine, whereas tryptophan was not associated with any of these parameters. This may indicate that kynurenine and QUIN are not causative of cardiac remodeling but may constitute surrogates of accelerated tryptophan degradation. In fact, also other elements of the tryptophankynurenine pathway, such as 3-hydroxykynurenine, quinolinate phosphoribosyl transferase or nicotinamide adenine dinucleotide phosphate oxidase, are linked with pro-inflammatory and cytotoxic effects $[6,9,38]$. Conversely, our observations could be explained by shared pathophysiological processes between cardiac remodeling and stimulated tryptophane-kynurenine pathway, such as atherosclerosis.

Limitations of our study include its cross-sectional character, which precludes definite conclusions on causeeffect relationships. Because of the single-center design, our results may not be generalizable to other populations. Moreover, because of the unavailability of cortisol levels in the studied cohort, we could not investigate a potential association between cortisol concentrations and parameters of the tryptophan-kynurenine pathway.

In conclusion, we found independent relationships between the tryptophan metabolites kynurenine and QUIN with echocardiographic parameters of cardiac remodeling. In particular, the independent associations of QUIN with LVMI, LV hypertrophy, LAVI, NT-proBNP and $\mathrm{E} / \mathrm{e}^{\prime}$ were striking. Our report may lead attention towards the previously underrecognized link between low-grade inflammation, activation of the tryptophan-kynurenine pathway and cardiac remodeling in pHPT. Our study may stimulate research on potential cardiovascular effects of tryptophan metabolites, particularly QUIN.

Acknowledgments: We thank the Laboratory of the Division of Endocrinology and Metabolism for its work and support to the present research. Probes used for the present analyses were partly provided by the Biobank Graz.

Author contributions: All the authors have accepted responsibility for the entire content of this submitted manuscript and approved submission.

Research funding: The study was funded by the Austrian National Bank (Jubilaeumsfond: project no. 14621) and by the Austrian Society for Bone and Mineral Research (FelixBronner Grant 2014, Project Prize 2014).
Employment or leadership: None declared.

Honorarium: None declared.

Competing interests: The funding organizations played no role in the study design; in the collection, analysis and interpretation of data; in the writing of the report; or in the decision to submit the report for publication.

\section{References}

1. McMahon DJ, Carrelli A, Palmeri N, Zhang C, DiTullio M, Silverberg SJ, et al. Effect of parathyroidectomy upon left ventricular mass in primary hyperparathyroidism: a meta-analysis. J Clin Endocrinol Metab 2015;100:4399-407.

2. Yu N, Donnan PT, Flynn RW, Murphy MJ, Smith D, Rudman $A$, et al. Increased mortality and morbidity in mild primary hyperparathyroid patients. The parathyroid epidemiology and audit research study (PEARS). Clin Endocrinol (Oxf) 2010;73:30-4.

3. Clifton-Bligh PB, Nery ML, Supramaniam R, Reeve TS, Delbridge L, Stiel JN, et al. Mortality associated with primary hyperparathyroidism. Bone 2015;74:121-4.

4. Stefenelli T, Abela C, Frank H, Koller-Strametz J, Globits S, Bergler-Klein J, et al. Cardiac abnormalities in patients with primary hyperparathyroidism: implications for follow-up. J Clin Endocrinol Metab 1997;82:106-12.

5. Mangge H, Stelzer I, Reininghaus EZ, Weghuber D, Postolache TT, Fuchs D. Disturbed tryptophan metabolism in cardiovascular disease. Curr Med Chem 2014;21:1931-7.

6. Wang Q, Zhang M, Ding Y, Wang Q, Zhang W, Song P, et al. Activation of NAD(P)H oxidase by tryptophan-derived 3-hydroxykynurenine accelerates endothelial apoptosis and dysfunction in vivo. Circ Res 2014;114:480-92.

7. Guillemin GJ. Quinolinic acid, the inescapable neurotoxin. FEBS J 2012;279:1356-65.

8. Pemberton LA, Kerr SJ, Smythe G, Brew BJ. Quinolinic acid production by macrophages stimulated with IFN-gamma, TNF-alpha, and IFN-alpha. J Interferon Cytokine Res 1997;17:589-95.

9. Maldonado PD, Molina-Jijon E, Villeda-Hernandez J, GalvanArzate S, Santamaria A, Pedraza-Chaverri J. NAD(P)H oxidase contributes to neurotoxicity in an excitotoxic/prooxidant model of Huntington's disease in rats: protective role of apocynin. J Neurosci Res 2010;88:620-9.

10. Lugo-Huitron R, Ugalde Muniz P, Pineda B, Pedraza-Chaverri J, Rios C, La Perez-de Cruz V. Quinolinic acid: an endogenous neurotoxin with multiple targets. Oxid Med Cell Longev 2013;2013:104024.

11. Pawlak K, Mysliwiec M, Pawlak D. Hyperhomocysteinemia and the presence of cardiovascular disease are associated with kynurenic acid levels and carotid atherosclerosis in patients undergoing continuous ambulatory peritoneal dialysis. Thromb Res 2012;129:704-9.

12. Pawlak K, Mysliwiec M, Pawlak D. Haemostatic system, biochemical profiles, kynurenines and the prevalence of cardiovascular disease in peritoneally dialyzed patients. Thromb Res 2010;125:e40-5.

13. Pedersen ER, Tuseth N, Eussen, Simone JP, Ueland PM, Strand E, et al. Associations of plasma kynurenines with risk of acute 
myocardial infarction in patients with stable angina pectoris. Arterioscler Thromb Vasc Biol 2015;35:455-62.

14. Eussen SJ, Ueland PM, Vollset SE, Nygard O, Midttun O, Sulo G, et al. Kynurenines as predictors of acute coronary events in the Hordaland Health Study. Int J Cardiol 2015;189:18-24.

15. Ogard CG, Engelmann MD, Kistorp C, Nielsen SL, Vestergaard H. Increased plasma $\mathrm{N}$-terminal pro-B-type natriuretic peptide and markers of inflammation related to atherosclerosis in patients with primary hyperparathyroidism. Clin Endocrinol (Oxf) 2005;63:493-8.

16. Christensen MH, Fenne IS, Nordbo Y, Varhaug JE, Nygard KO, Lien EA, et al. Novel inflammatory biomarkers in primary hyperparathyroidism. Eur J Endocrinol 2015;173:9-17.

17. Almqvist EG, Bondeson AG, Bondeson L, Svensson J. Increased markers of inflammation and endothelial dysfunction in patients with mild primary hyperparathyroidism. Scand J Clin Lab Invest 2011;71:139-44.

18. Christensen MH, Pedersen EK, Nordbo Y, Varhaug JE, Midttun 0 , Ueland PM, et al. Vitamin B6 status and interferon-gammamediated immune activation in primary hyperparathyroidism. J Intern Med 2012;272:583-91.

19. Meinitzer A, Tomaschitz A, Pilz S, Truber M, Zechner G, Gaksch $M$, et al. Development of a liquid chromatography-mass spectrometry method for the determination of the neurotoxic quinolinic acid in human serum. Clin Chim Acta 2014;436:268-72.

20. Tomaschitz A, Fahrleitner-Pammer A, Pieske B, Verheyen N, Amrein K, Ritz E, et al. Effect of eplerenone on parathyroid hormone levels in patients with primary hyperparathyroidism: a randomized, double-blind, placebo-controlled trial. BMC Endocr Disord 2012;12:19.

21. Tomaschitz A, Verheyen N, Meinitzer A, Pieske B, Belyavskiy E, Brussee $\mathrm{H}$, et al. Effect of eplerenone on parathyroid hormone levels in patients with primary hyperparathyroidism: results from the EPATH randomized, placebo-controlled trial. J Hypertens 2016;34:1347-56.

22. Herve C, Beyne P, Jamault H, Delacoux E. Determination of tryptophan and its kynurenine pathway metabolites in human serum by high-performance liquid chromatography with simultaneous ultraviolet and fluorimetric detection. J Chromatogr B Biomed Appl 1996;675:157-61.

23. Verheyen N, Fahrleitner-Pammer A, Pieske B, Meinitzer A, Belyavskiy E, Wetzel J, et al. Parathyroid hormone, aldosteroneto-renin ratio and fibroblast growth factor-23 as determinants of nocturnal blood pressure in primary hyperparathyroidism: the eplerenone in primary hyperparathyroidism trial. J Hypertens 2016;34:1778-86.

24. Lang RM, Badano LP, Mor-Avi V, Afilalo J, Armstrong A, Ernande $L$, et al. Recommendations for cardiac chamber quantification by echocardiography in adults: an update from the American society of echocardiography and the European association of cardiovascular imaging. J Am Soc Echocardiogr 2015;28:1-39.e14.

25. Nagueh SF, Smiseth OA, Appleton CP, Byrd BF, Dokainish H, Edvardsen $\mathrm{T}$, et al. Recommendations for the evaluation of left ventricular diastolic function by echocardiography: an update from the American society of echocardiography and the Euro- pean association of cardiovascular imaging. J Am Soc Echocardiogr 2016;29:277-314.

26. Devaux B, Scholz D, Hirche A, Klovekorn WP, Schaper J. Upregulation of cell adhesion molecules and the presence of low grade inflammation in human chronic heart failure. Eur Heart J 1997;18:470-9.

27. Bekwelem W, Lutsey PL, Loehr LR, Agarwal SK, Astor BC, Guild C, et al. White blood cell count, C-reactive protein, and incident heart failure in the atherosclerosis risk in communities (ARIC) study. Ann Epidemiol 2011;21:739-48.

28. Kaptoge S, Di Angelantonio E, Lowe G, Pepys MB, Thompson SG, Collins $\mathrm{R}$, et al. C-reactive protein concentration and risk of coronary heart disease, stroke, and mortality: an individual participant meta-analysis. Lancet 2010;375:132-40.

29. Watts SW, Shaw S, Burnett R, Dorrance AM. Indoleamine 2,3-diooxygenase in periaortic fat: mechanisms of inhibition of contraction. Am J Physiol Heart Circ Physiol 2011;301:H1236-47.

30. Gao X, Xu X, Pang J, Zhang C, Ding JM, Peng X, et al. NMDA receptor activation induces mitochondrial dysfunction, oxidative stress and apoptosis in cultured neonatal rat cardiomyocytes. Physiol Res 2007;56:559-69.

31. Meng L, Zhang Z, Xu K, Qi G. HIV-1 gp120 induces autophagy in cardiomyocytes via the NMDA receptor. Int J Cardiol 2013;167:2517-23.

32. Wang Q, Liu D, Song P, Zou MH. Tryptophan-kynurenine pathway is dysregulated in inflammation, and immune activation. Front Biosci (Landmark Ed) 2015;20:1116-43.

33. Fernandes A, Landeira-Fernandez AM, Souza-Santos P, CarvalhoAlves PC, Castilho RF. Quinolinate-induced rat striatal excitotoxicity impairs endoplasmic reticulum Ca2+-ATPase function. Neurochem Res 2008;33:1749-58.

34. Pawlak K, Mysliwiec M, Pawlak D. Kynurenine pathway - a new link between endothelial dysfunction and carotid atherosclerosis in chronic kidney disease patients. Adv Med Sci 2010;55:196-203.

35. Ball HJ, Yuasa HJ, Austin CJ, Weiser S, Hunt NH. Indoleamine 2,3-dioxygenase-2; a new enzyme in the kynurenine pathway. Int J Biochem Cell Biol 2009;41:467-71.

36. Niimi S, Nakamura T, Nawa K, Ichihara A. Hormonal regulation of translatable mRNA of tryptophan 2,3-dioxygenase in primary cultures of adult rat hepatocytes. J Biochem 1983;94:1697-706.

37. Gianotti L, Tassone F, Pia A, Bovio S, Reimondo G, Visconti G, Terzolo M, Borretta G. May an altered hypothalamo-pituitaryadrenal axis contribute to cortical bone damage in primary hyperparathyroidism? Calcif Tissue Int 2009;84:425-9.

38. Ishidoh K, Kamemura N, Imagawa T, Oda M, Sakurai J, Katunuma N. Quinolinate phosphoribosyl transferase, a key enzyme in de novo $\mathrm{NAD}(+)$ synthesis, suppresses spontaneous cell death by inhibiting overproduction of active-caspase-3. Biochim Biophys Acta 2010;1803:527-33.

Supplemental Material: The online version of this article (DOI: 10.1515/cclm-2016-1159) offers supplementary material, available to authorized users. 